\title{
Shear Stress in High-Pressure Torsion and Vickers Hardness of Au-Co Alloys
}

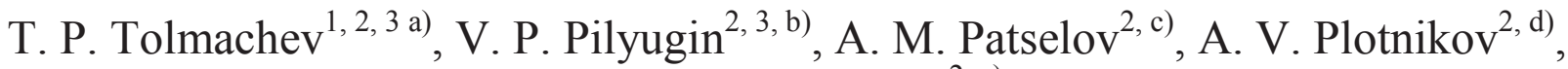 \\ and R. V. Churbaev' ${ }^{2,}$ ) \\ ${ }^{1}$ Institute of Engineering Science, Ural Branch of the Russian Academy of Sciences, 34 Komsomolskaya St., \\ Ekaterinburg, 620049, Russia \\ ${ }^{2}$ M. N. Miheev Institute of Metal Physics, Ural Branch of the Russian Academy of Sciences, 18 S. Kovalevskoy St., \\ 620108, Ekaterinburg, Russia \\ ${ }^{3}$ B. N. Yeltsin Ural Federal University, 19 Mira St., Ekaterinburg, 620002, Russia \\ ${ }^{\text {a)} C o r r e s p o n d i n g ~ a u t h o r: ~ t o l m a c h e v @ i m p . u r a n . r u ~}$ \\ ${ }^{b)}$ pilyugin@imp.uran.ru \\ c) patselov@imp.uran.ru \\ d)plotnikov@imp.uran.ru \\ e)churbaev@imp.uran.ru
}

\begin{abstract}
Alloys of the Au-Co system characterized by restricted solubility of the constituents were produced by shear deformation under high quasi-hydrostatic pressure. Resistance to shear strain was recorded during mechanical fusion. The forming pressure dependence of shear stress during deformation has been revealed, with the form of the curve remaining qualitatively unchanged. The form of the curve for the joint deformation of the Au-Co constituents differs from the curve recorded during the deformation of one constituent, namely gold. In the former case there are stages of sharp increase, retardation, and attainment of saturation, whereas in the latter case there is a sharp increase followed by attainment of saturation. This indicates the appearance of the deformation interaction of the constituents, which manifests itself in a prolonged increase in shear stress with an increase in strain, as distinct from the case of a single element, where there occur only structural changes caused by torsion under pressure. After the samples had been made, their Vickers hardness was measured, which demonstrates practically equal values of the hardness of the Au-Co alloys produced under different pressures.
\end{abstract}

\section{INTRODUCTION}

The Au-Co system is characterized by restricted solubility, practically zero at room temperature $[1,2]$. According to the Miedema semi-empirical model, the constituents of the system under study have a positive enthalpy of solution [3]. Besides, gold and cobalt essentially differ in their physical and mechanical characteristics $[2,4]$. Systems with restricted solubility, such as Au-Co, are of interest as materials with controlled structure and properties due to the fact that metastable phases, if they are obtained, can be purposely affected, with corresponding structures and/or phases resulting from affecting. Among the methods for forming metastable phases there are ultrarapid melt quenching [5, 6], irradiation with high-energy particles [7], mechanical fusion [8], and severe plastic deformation $[9,10]$.

The method of torsion under high quasi-hydrostatic pressure in a Bridgman chamber [11, 12], originally belonging to methods of severe plastic deformation [12,13], enables us also to perform mechanical fusion [14]. The resulting alloys are in a monolithic bulk form, suitable for further examination. Besides, this method allows us to vary the processing parameters, thus affecting the characteristics of the structure and properties at the stage of obtaining a finished sample. Most of the studies of the properties of mechanically synthesized alloys deal with the

Mechanics, Resource and Diagnostics of Materials and Structures (MRDMS-2020) AIP Conf. Proc. 2315, 040046-1-040046-4; https://doi.org/10.1063/5.0036671 Published by AIP Publishing. 978-0-7354-4057-9/\$30.00 
post-deformation period; however, there are methods for in-situ behavior monitoring during processing, e.g. [15, 16]. Among them there is shear stress measurement during deformation [16-18].

The aim of this study is to monitor the mechanical behavior of the components of a system of restricted solubility, practically zero at the processing temperature in this study, by resistance to deformation during mechanical fusion depending on the forming pressure. To obtain information on subsequent mechanical behavior, we measured the Vickers hardness of the obtained alloys.

\section{MATERIALS AND METHODS}

Cobalt with a purity of $99.60 \%$ and gold with a purity of $99.99 \%$ in the initial state were in the powder form, with a particle size of $\sim 50 \mu \mathrm{m}$ and $\sim 300 \mu \mathrm{m}$, respectively. The powdery constituents were mixed in equiatomic proportion and then shear-deformed under high pressure on rotating Bridgman anvils (the method is most widely known as high-pressure torsion, HPT) at room temperature. The anvil type is quasi-constrained [12], the working area diameter is $5 \mathrm{~mm}$, and the anvil material is VK-6 with a hardness of $92 \mathrm{HRC}$.

Severe plastic deformation of the powder mixtures was performed under different pressures, namely $6,8,10$, and $12 \mathrm{GPa}$. The mechanically fused alloy samples were disk-shaped, $5 \mathrm{~mm}$ in diameter and about $0.1 \mathrm{~mm}$ thick. Resistance to torsional deformation depending on the angle of rotation was measured in situ under pressure, with the use of an ATsDS-1I-1 electronic dynamometer. Shear stress $\tau$ for the values of the anvil radius $R=2.5 \mathrm{~mm}$ was computed by the formula found in [16]. Hardness was measured by the Vickers method with a force of $0.49 \mathrm{~N}$ in a PMT-3 hardness tester.

\section{RESULTS}

When gold is deformed without cobalt, shear stress sharply increases immediately after the start of the torsion procedure, and it reaches about $2.8 \mathrm{GPa}$ (Fig. 1). Thereafter, the material ceases to show any increase in shear strain resistance. This dependence was recorded at a pressure of $8 \mathrm{GPa}$.

In the case of a gold-cobalt mixture with an equivalent ratio of the components, there is a smoother increase in the torsional moment instead of its sharp increase (Fig. 1). At about $2.8 \mathrm{GPa}$, the shear stress does not stop growing, it continues growing for a fairly long time, within which the anvils manage to perform 8 to 10 full turns. It is not before the anvil makes more than 10 turns that the saturation state is reached.

Deformation at lower pressure, about $6 \mathrm{GPa}$, yields slightly lower values for the whole dependence of shear stress on the number anvil turns; however, saturation is attained at about $3.2 \mathrm{GPa}$, this being higher than in the case of pure gold.

The pressure increase to $12 \mathrm{GPa}$ leads to even higher values of the strain dependence of shear stress, both on the whole and at the attainment of saturation of the increase in shear strength.

In the case of the mixture, at all processing pressures, the shear stress curves are characterized by a practically equal number of anvil turns after which the forming alloy resists shear strain, i.e. about 8 to 10 turns.

At the same time, it should be taken into account that the strain dependences of shear stress represent the integral processes of structural and, for mixtures, phase changes. This is particularly inherent in the initial stage, when the ultrafine-grain state can already be attained on the sample periphery, but not yet in the sample center. Naturally, after a large number of anvil turns is attained, the structural and phase transformations typical of the periphery occur in the sample center, too. In this case, there is a plateau in the shear strain resistance.

The Vickers hardness measurement has shown that the hardness is distributed on the surfaces of all the samples in the range of values of about $1.0 \mathrm{GPa}$. This reflects that the processes increasing shear strain resistance attain saturation. The diagram shown in Fig. 2 presents hardness values averaged over the surface for each alloy produced under different pressures and for pure constituents processed separately. The hardness values of the Au-Co alloys do not differ from each other and from those of cobalt (within the measurement error).

Comparing the data on the mechanical behavior of the Au-Co alloys during and after their formation, we can conclude that, after the alloys have been produced, processes occur which result in that the properties of the nearsurface regions, at least accessible for penetration of a diamond indenter, level off and become practically equal to the values of the hardness of cobalt, consolidated from powder and deformed. The hardness of deformed gold is relatively low as compared to that of its alloys with cobalt (Fig. 2). Herewith, the difference from the properties in dynamics, probably, indicates the processes of precipitation of the second phase in the gold-cobalt alloys, which 
hardens the alloy, and processes related to grain growth in gold, which occur after the samples have been produced, during their aging at room temperature for 24 to 96 hours.

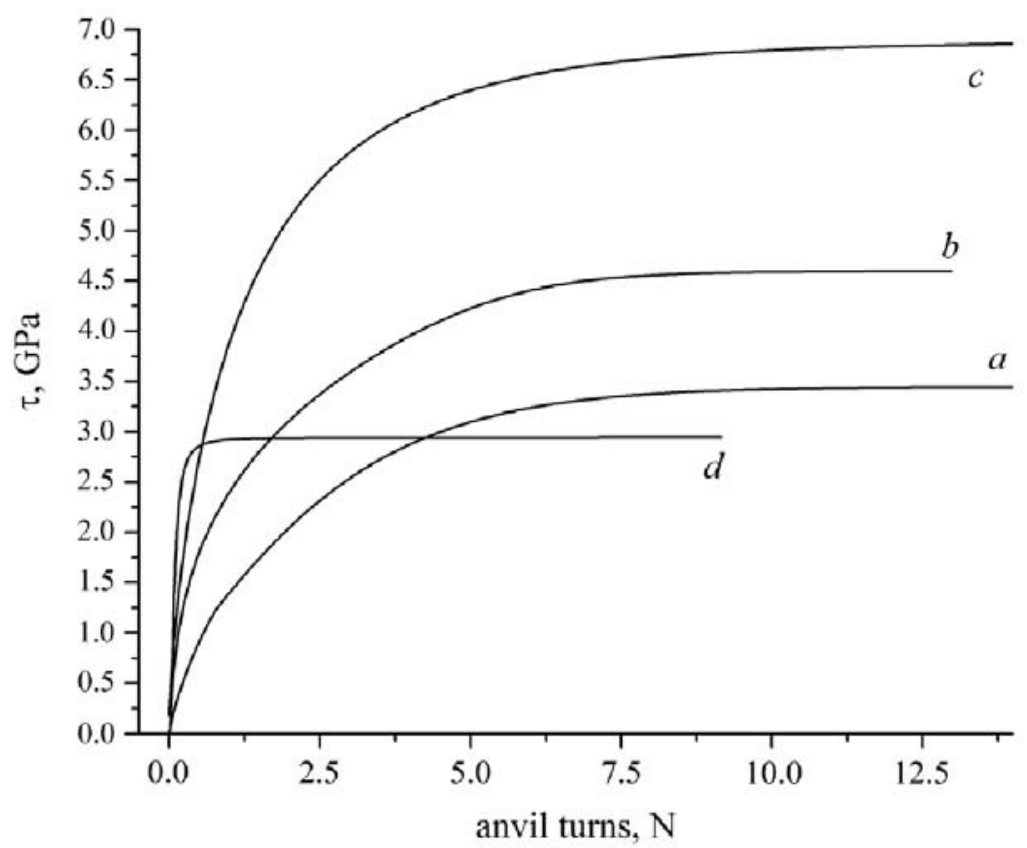

FIGURE 1. In-situ shear stresses as dependent on the value of large plastic strain: for the Au-Co equiatomic mixture under a processing pressure of $6 \mathrm{GPa}(\mathrm{a}), 8 \mathrm{GPa}(\mathrm{b}), 12 \mathrm{GPa}(\mathrm{c})$ and for gold (d)

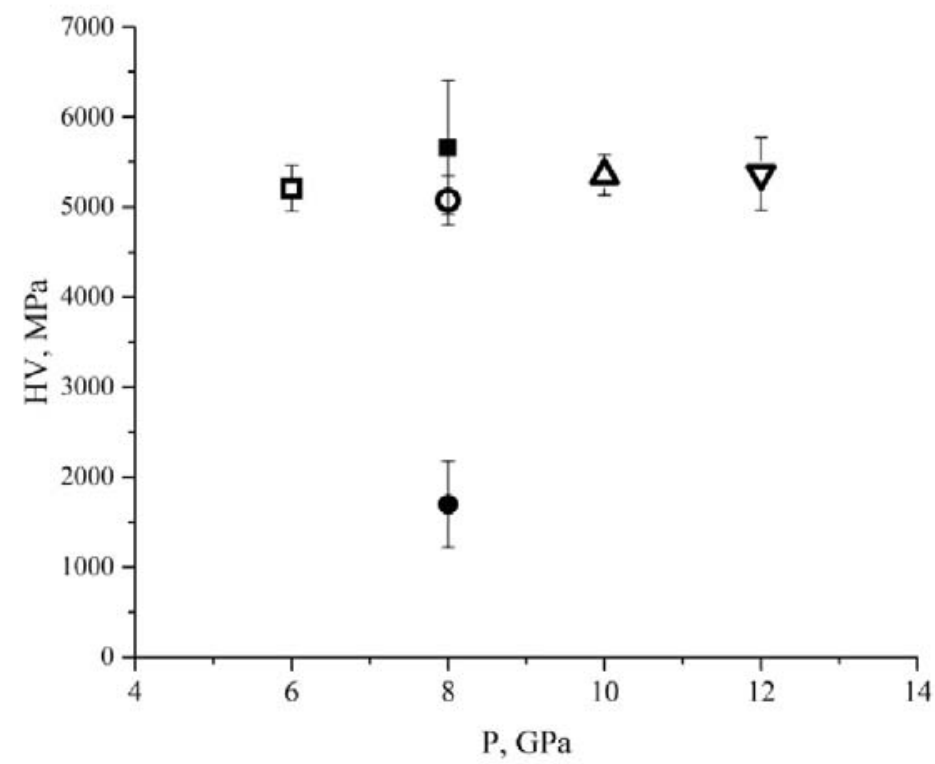

FIGURE 2. Vickers hardness values for Au-Co alloys produced by large plastic deformation under the following pressures: $6 \mathrm{GPa}(\square), 8 \mathrm{GPa}(\circ), 10 \mathrm{GPa}(\Delta), 12 \mathrm{GPa}(\nabla)$, and for gold $(\bullet)$ and cobalt $(\boldsymbol{\bullet})$ 


\section{CONCLUSION}

Mechanical fusion of mutually insoluble constituents of the Au-Co system from their equiatomic powder mixture was performed by severe plastic torsion deformation under high pressure at room temperature. The processing pressure was varied with an increment of $2 \mathrm{GPa}$, starting from $6 \mathrm{GPa}$ and finishing with $12 \mathrm{GPa}$. The monitoring of resistance to deformation has shown a pressure dependence of most alloys. A comparison with a pure constituent has revealed the presence of a curve portion responsible for a smooth increase in shear stress with the value of strain between a sharp, almost linear, growth and attainment of saturation, this being attributable to the processes of straininduced interaction of the constituents. The hardness measurements of the obtained alloys have shown practically identical hardness values, close to those for deformed pure cobalt and significantly exceeding the values for deformed gold; when comparing with the data obtained in dynamics, this reflects post-deformation processes which may be due to a decay of the metastable phase of the solid solution and an increase in the volume fraction of the phase associated with cobalt in the alloy samples.

\section{ACKNOWLEDGMENTS}

The study was performed under a state assignment and with a financial support from the RFBR, project No. 1932-60039. The materials were produced and processed on the basis of the IMP UB RAS, Ekaterinburg.

\section{REFERENCES}

1. H. Okamoto, T. B. Massalski, M. Hasebe, and T. Nishizawa, Bull. Alloy Phase Diagrams 6(5), 449-454 (1985).

2. O. M. Barabash and Yu. N. Koval, Structure and Properties of Metals and Alloys, (Naukova Dumka, Kiev, 1986).

3. A. R. Miedema, P. F. de Chatel, and F. R. de Boer, Physica B+C 100(1), 1-28 (1980).

4. M. E. Drits (ed.), Properties of Elements: Handbook ed. by (Metallurgiya, Moscow, 1985).

5. J. Yin, G. Chen, H. Tang, and X. Qu, Int. J. Refract. Met. Hard Mater. 92, 105250 (2020).

6. X. Meng, D. Zhang, W. Zhang, C. Qiu, G. Liang, and J. Chen, J. Alloy. Compd. 819, 152990 (2020).

7. L. T. Belkacemi, E. Meslin, B. Décamps, J.-P. Crocombette, O. Tissot, T. Vandenberghe, P. Desgardin, T. Sauvage, and C. Berthier, Scr. Mater. 188, 169-173 (2020).

8. A. Tejeda-Ochoa, N. Kametani, C. Carreño-Gallardo, J. E. Ledezma-Sillas, N. Adachi, Y. Todaka, and J. M. Herrera-Ramirez, Powder Technology 374, 348-352 (2020).

9. A. V. Dobromyslov, N. I. Talutsб and V. P. Pilyugin, Int. J. Refract. Met. Hard Mater. 93, 105354 (2020).

10. J. Sun, Q. Ke, and W. Chen, J. Mater. Process. Tech. 264, 119-128 (2019).

11. P. W. Bridgman, Phys. Rev. 48, 825-847 (1935).

12. A. P. Zhilyaev and T. G. Langdon, Prog. Mater. Sci. 53(6), 893- 979 (2008).

13. A. M. Glezer, E. V. Kozlov, N. A. Koneva, N. A. Popova, and I. A. Kurzina, Plastic Deformation of Nanostructured Materials (CRC Press, Boca Raton, 2017).

14. V. A. Teplov, V. P. Pilugin, V. S. Gaviko, and E. G. Chernyshov, Phil. Mag. B 68(6), 877-881 (1993).

15. R. Nomura and K. Uesugi, Rev. Sci. Instrum. 87(4), 046105 (2016).

16. L. C. Towle and R. E. Riecker, Science 163, 41-47 (1969).

17. R. V. Sundeev, A. V. Shalimova, A. M. Glezer, E. A. Pechina, M. V. Gorshenkov, and G. I. Nosova, Mat. Sci. Eng. A 679, 1-6 (2017).

18. R. V. Sundeev, A. V. Shalimova, N. N. Sitnikov, O. P. Chernogorova, A. M. Glezer, M. Yu. Presnyakov, I. A. Karateev, E. A. Pechina, and A. V. Shelyakov, J. Alloys Compd. 845, 156273 (2020). 